\title{
PROBLEMÁTICA DEL TRÁNSITO BINACIONAL DE UNA NACIÓN TERRITORIALMENTE DIVIDIDA: EL CASO DE LOS TOHONO O'ODHAM
}

\author{
Brenda Alicia Gutiérrez Cañez ${ }^{23}$ \\ María Auxiliadora Moreno Valenzuela ${ }^{24}$
}

Sumario: I. Introducción. II.La división del territorio de la Nación.III. Problemas del tránsito entre Sonora y Arizona. IV.Conclusiones.

\section{Resumen}

En el presente trabajo se analiza el hecho de que la Nación Tohono O'odham haya quedado dividida territorialmente al perder México parte de su territorio, lo cual plantea para su población, una serie de problemas como lo son la división de su territorio, la pérdida de la identidad cultural, la separación de familias, problemas que se enmarcan en la imposibilidad de transitar libremente entre la frontera Arizona - Sonora.

Palabras claves: Nación Tohono O'odham, rituales y tradiciones, territorio estadounidense y mexicano

\section{Abstract}

This paper discusses the fact that the Tohono O'odham nation has been divided territorially after losing Mexico part of its territory, which raises to its population, a number of problems such as the division of its territory, the loss of cultural identity, the separation of families, problems that are part of the impossibility to travel freely between Arizona - Sonora border.

Key words:Tohono O'odham Nation, rituals and traditions, American and Mexican territory

\footnotetext{
${ }^{23}$ Estudiante de la Licenciatura en Derecho Auxiliar de Investigación bajo la dirección y asesoría de la Dra. María Auxiliadora Moreno Valenzuela.

${ }^{24}$ Profesora Investigadora de Tiempo Completo del Departamento de Derecho de la Universidad de Sonora. Unidad Regional Centro.
} 


\section{Introducción}

Se busca conocer la problemática relacionada con el ejercicio de derechos de los integrantes de la nación Tohono O'odham especificamente el derecho a conservar y preservar su identidad cultural, ejercicio que se ve limitado en tanto que se impide el libre tránsito su territorio que, como veremos se encuentra dividido geográficamente por la frontera, aunque ellos siguen conservando una sola identidad como nación.

\section{La división del territorio de la Nación Tohono O'odham}

La nación Tohono O'odham formó parte del pueblo Pima hasta el período virreinal, en que la colonización y conversión los dividió. El proceso de aculturación de los Tohono O'odham fue mucho más lento y tardío que el de otros grupos indígenas, comenzando hasta el siglo XVIII de la mano de misioneros como el padre Kino y Salmair. ${ }^{25}$

El tratado de Guadalupe-Hidalgo ${ }^{26}$, significó la pérdida de territorios para nuestro país y la división del territorio de la NaciónTohono O'odham. Como señala Salas Quintanal, "Con el desplazamiento de la frontera, MéxicoEstados Unidos hacia el sur en 1848 comienza la fragmentación territorial, no sólo de los grupos indígenas asentados en la zona, sino también de familias y bandas que fueron divididas entre dos nacionalidades". ${ }^{27}$ Con la división territorial la mayor parte de los integrantes de la Nación O'odham quedaron ubicados del lado estadounidense, manteniéndose sin embargo, su identidad

\footnotetext{
${ }^{25}$ Castillo, Guillermo, "Lasveredas entre el desierto y la ciudad". reconfiguración de la identidad en el proceso histórico de cambio de los tohono ó odham. Tesis para optar por el grado de doctor en antropología http://www.naya.org.ar/tesis/Guillermo_Castillo/

${ }^{26}$ El tratado se aprobó en el senado con fecha el 10 de Marzo de 1848.

${ }^{27}$ Salas, Quintanal, Hernán J., "La "gente del desierto" en el norte de Sonora", UNAM, Culturales, vol. II, núm. 3, enero-junio, 2006, pp. 9-31, Red de Revistas Científicas de América Latina, el Caribe, España y Portugal,http://www.redalyc.org/pdf/694/69420302.pdf.
} 
de Nación, al conservar y fortalecer los lazos entre ellos, a través de ambas fronteras hasta el día de hoy.

Castillo Ramírez, destaca como, en el reforzamiento de estos lazos contribuyeron en gran medida el hecho de estar ubicados los integrantes de la Nación, en ambas partes de la zona fronteriza; asi como el que los gobernantes de la Nación Tohono O'odham, cuyos poderes están asentados en el territorio estadounidense validaran o ratificaran la pertenencia a la Nación de aquéllos miembros que habían quedado establecidos en territorio mexicano.

No obstante, en este sentido es interesante destacar que, a diferencias de otros experiencia similares de resurgimiento de la etnicidad, aquí jugó un papel preponderante la condición geográfica (ser zona fronteriza) y la historia de los O'odham, pues particularmente en el caso de los O'odham de Sonora se reforzaron los lazos con los miembros del grupo étnico en Arizona a través de las acciones de la Nación Tohono O'odham (NTO) para incorporar dentro de sus registros a aquellos O’odham que estaban más allá de la frontera en México. ${ }^{28}$

Algunas de las acciones que se siguen realizando en la actualidad para rescatar la cultura de la Nación en México son entre otras, la realización de rituales y ceremonias tradicionales tales como los rituales que realizan en el marco de las fiestas de San Francisco y los rituales de la lluvia que se realizan generalmente en México y en EEUU el Anual Rodeo and fair y wa:k-puwowow ${ }^{29}$

La Nación Tohono O'odham en México se encuentra localizada al noroeste del estado de Sonora, ocupan casi 83000 hectáreas del valle de

\footnotetext{
${ }^{28}$ Castillo, Guillermo op cit., nota 2

${ }^{29}$ Arizona Guide http://www.arizonaguide.com/things-to-do/tohono-oodhamnation-sells-az
} 
Altar, divididos en seis pequeñas localidades las cuales son: Pozo Prieto, Las Norias y San Francisquito del municipio de Caborca; Quitovac, del municipio de Puerto Peñasco; Pozo Verde, municipio de Saric, y El Bajío, municipio de Altar, allí mismo conviven con los habitantes de este municipio ${ }^{30}$.

En Estados Unidos de Norteamérica, las tierras de la nación están localizadas en el sur central de Arizona. Siendo Sells la capital de la Nación Tohono O'odham en dicho territorio. ${ }^{31}$ Cuatro de sus Distritos contienen más de 2.7 millones de acres comenzando por el sur de Casa Grande y partes del condado Pinal, Pima y Maricopa antes de continuar al sur de México.

San Xavier es el segundo territorio más grande y contiene 71,095 acres al sur de la ciudad de Tucson. Pequeñas parcelas incluyen el distrito de san Lucy localizado cerca de Gila Bend y Florence Village, el cual es localizado cerca de Florence. $^{32}$

III. Problemas del tránsito de los integrantes de la Nación, entre Sonora y Arizona.

El tratado de Guadalupe -Hidalgo no incorporó a la Nación Tohono O'odham como tal, solamente hizo la distinción en cuanto a derechos de propiedad territorial y de ciudadanía que tendrían los mexicanos que quedaron asentados en territorio estadounidense y territorio mexicano, a partir de la división territorial $^{33}$. Amnistía Internacional señala que "El Tratado de GuadalupeHidalgo que estableció la frontera entre México y EEUU en 1853 afirmó los derechos de los pueblos indígenas que viven a lo largo de la frontera a

\footnotetext{
30"La medicina tradicional de los pueblos indígenas de México", Biblioteca Digital de la Medicina Tradicional Mexicana

http://www. medicinatradicionalmexicana. unam. $m x /$ pueblos. php?l=2\&t=papago\& $m o=\& d e m a n d a=\& o r d e n=\& v=$

${ }^{31}$ Sitio web de la Nación Tohono O'odham http://www.tonationnsn.gov/history_culture.aspx

${ }^{32}$ idem

${ }^{33}$ Soberanes, José Luis, Vega, Juan Manuel, "El tratado de Guadalupe Hidalgo en su Sesquicentenario" http://biblio.juridicas.unam.mx/libros/1/166/3.pdf Articulo VIII, IX y X del tratado.
} 
mantener sus tierras, cultura, y religión. Sin embargo, no incluyó ninguna referencia explícita sobre los derechos indígenas de cruzar la frontera."34

En el 2009, la Nación Tohono O'odham firmó un acuerdo con el Departamento de Seguridad Interna del país de Estados Unidos, para que los miembros de la nación pudieran reingresar sin problemas al país, mediante una Ley denominada para el Hemisferio Occidental la cual contempló la emisión de la Tarjeta Incremental de Indígena Nativo Americano. Esta ley trataba de resolver la problemática de los miembros de la Nación Tohono O'odham que salían rumbo a México ya sea a visitar a sus familiares o realizar las ceremonias propias de la comunidad pero, no contemplo la situación de los integrantes de los Tohono O'odham establecidos en México que requerían ingresar a los Estados Unidos, para el cumplimiento de las mismas tradiciones o el visitar a sus familiares.

La Tarjeta de identificación Tribal que se les otorga a los miembros nacidos en Territorio Estadounidense, señala las siglas del Distrito que la expide, mientras que las tarjetas para los miembros nacidos en México indican "ND" que significa "no distrito".

En los últimos años, la política fronteriza ha llegado a afectar a los O'odham en muchos aspectos, ya que las leyes de inmigración impiden que el O'odham pueda cruzar libremente. De hecho, la frontera México-Estados Unidos se ha convertido en una limitante para que puedan realizar sus ritos y tradiciones necesarios para el mantenimiento de su identidad cultural.

“...Del mismo modo, en muchas ocasiones las Aduanas de Estados Unidos han impedido a los Tohono O'odham el transporte de materias primas y mercancías esenciales de su espiritualidad, la economía y la cultura tradicional. Los funcionarios de fronteras

\footnotetext{
${ }^{34}$ Amnistía Internacional, En Terreno hostil: las violaciones a los derechos humanos en la aplicación de las leyes de inmigración en el suro este de estados unidos pp. 27 capítulo 3: abusos contra los pueblos indígenashttp://www.amnestyusa.org/pdfs/informedeinmigracion.pdf
} 
también se han confiscado bienes culturales y religiosos, como las plumas de las aves comunes, hojas de pino o hierba dulce." ${ }^{35}$.

Amnistía Internacional ha documentado esta problemática de tránsito de los integrantes de la Nación, a partir de entrevistas realizadas a miembros de los Tohono O'odham, destacando entre otras, las siguientes circunstancias: a) que los integrantes de la Border Patrol no respetan las tarjetas que otorga la tribu a los miembros de la misma, impidiéndoles el libre acceso a su territorio en Estados Unidos. b) que los agentes violentan física y verbalmente a las personas miembros de la tribu que intentan cruzar amparados en su tarjeta que los identifica como miembros de la Nación Tohono Odham.

\section{Conclusiones}

La división territorial de la Nación Tohono O'odham ha implicado para su población la afectación de sus derechos, desde la división de su territorio hasta limitación de relacionarse con sus familiares que viven "al otro lado" (sea de México o de EEUU) y la preservación de su cultura y tradiciones.

Hasta hoy las leyes y convenios que se han acordado tanto entre la Nación Tohono O'odham con el gobierno norteamericano, como entre México y EEUU, para resolver esta situación no han dado resultado, primero porque no incorporan la necesidad de los miembros de la Nación asentados en el territorio mexicano, de trasladarse hacia el territorio estadounidense para la realización de sus ritos y ceremonias y visitar a sus familiares y segundo porque a pesar de existir el reconocimiento jurídico de las tarjetas que pretenden garantizar el reingreso a EEUU, estas no son respetadas por las autoridades migratorias.

\footnotetext{
${ }^{35}$ Sitio Web de la Nación Tohono O’odham Op cit., nota 8
} 
Bibliografía y Webgrafía:

AMNISTÍA INTERNACIONAL, En Terreno hostil: las violaciones a los derechos humanos en la aplicación de las leyes de inmigración en el suroeste de estados unidos pp. 27 capítulo 3: abusos contra los pueblos indígenas http://www.amnestyusa.org/pdfs/informedeinmigracion.pdf

ARIZONA GUIDEhttp://www.arizonaguide.com/things-to-do/tohono-oodhamnation-sells-az

BIBLIOTECA DIGITAL DE LA MEDICINA TRADICIONAL MEXICANA "La Medicina Tradicional de los Pueblos Indígenas de México", http://WwW. medicinatradicionalmexicana. unam. $m x /$ pueblos. php?l=2\&t=papago\& $m o=\& d e m a n d a=\& o r d e n=\& v=$

CASTILLO,RAMÍREZ, Guillermo, "LAS VEREDAS ENTRE EL DESIERTO Y LA CIUDAD". RECONFIGURACIÓN DE LA IDENTIDAD EN EL PROCESO HISTÓRICO DE CAMBIO DE LOS TOHONO O'ODHAM. TESIS PARA OPTAR POR EL GRADO DE DOCTOR EN ANTROPOLOGÍA

http://www.naya.org.ar/tesis/Guillermo_Castillo/

SALAS, QUINTANAL, Hernán J., "La "gente del desierto" en el norte de Sonora", UNAM, Culturales, vol. II, núm. 3, enero-junio, 2006, pp. 9-31, Red de Revistas Científicas de América Latina, el Caribe, España y Portugal,http://www.redalyc.org/pdf/694/69420302.pdf.

------op cit., nota 2

SITIO WEB DE LA NACION TOHONO O'ODHAM http://www.tonationnsn.gov/history_culture.aspx

idem

SOBERANES, José Luis, Vega, Juan Manuel, " el tratado de Guadalupe hidalgo en su sesquicentenario" http://biblio.juridicas.unam.mx/libros/1/166/3.pdf

Articulo VIII, IX y X del tratado op cit., nota 8 